Écrire

l'histoire

\section{Écrire l'histoire}

Histoire, Littérature, Esthétique

15 | 2015

La fin de l'histoire

\title{
D'un ton anti-apocalyptique : Après la fin du monde de Michaël Foessel et Récit d'un désastre de Michaël
}

Ferrier

\section{Catherine Coquio}

\section{(2) OpenEdition}

Journals

Édition électronique

URL : http://journals.openedition.org/elh/610

DOI : $10.4000 /$ elh.610

ISSN : 2492-7457

Éditeur

CNRS Éditions

\section{Édition imprimée}

Date de publication : 8 octobre 2015

Pagination : 119-128

ISBN : 978-2-271-08822-2

ISSN : 1967-7499

Référence électronique

Catherine Coquio, « D'un ton anti-apocalyptique : Après la fin du monde de Michaël Foessel et Récit d'un désastre de Michaël Ferrier », Écrire l'histoire [En ligne], 15 | 2015, mis en ligne le 08 octobre 2018, consulté le 23 septembre 2020. URL : http://journals.openedition.org/elh/610 ; DOI : https://doi.org/ 10.4000/elh.610 


\section{D'un ton anti- apocalyptique : Après la fin du monde de Michaël Foessel et Récit d'un désastre de Michaël Ferrier}

It is easier to imagine the end of the world than to imagine the end of capitalism.

Fredric JAMESON

(«Future City», New Left Review, n² 21, 2003)

L'année 2012, on s'en souvient, a été celle d'un festival d'apocalypses, et la fameuse prophétie maya $\mathrm{du}$ «Cinquième Soleil» n'y a joué le rôle que de signal déclencheur: les ingénieurs culturels pourvoyant aux joies troubles des nantis du monde étaient prêts depuis longtemps pour la fête posthumaine, et, avec cette mobilisation de l'eschatologie au service du divertissement de masse, une étape a dû être franchie dans la très riche histoire culturelle occidentale de l'apocalypse.
Celle-ci s'était depuis longtemps déjà installée dans le registre de "l'Apocalypse sans royaume». Cette formule utilisée par Günther Anders à propos de l'après-Hiroshima (La Menace nucléaire, 1981), actualisée par les penseurs du «catastrophisme éclairé» (Jean-Pierre Dupuy), l'a été autrement en littérature par d'innombrables fictions de la fin du monde inspirées par l'idée d'un temps historique devenu «délai» (Anders). Dans un livre récent de Jean-Paul Engé- 
libert, la lecture de plusieurs d'entre elles devient une alternative critique aux approches théoriques, qui tournent souvent à l'idéologie. Dans ces textes littéraires, on voit au contraire se former une «conscience tragique laïque», propre à une "humanité qui juge sa propre histoire», une fois celle-ci achevée. Sans prétendre détenir un discours de vérité, ces fictions "témoignent sans doute mieux que la théorie» de notre "grand désarroi", car un "bouleversement historique s'éprouve avant de se penser ${ }^{1} »$. Mais leur complexité nous fait aussi reposer la question politique d'Anders: "Comment faire mentir la prédiction et, depuis le délai, dans le temps qui nous est donné, ressusciter une histoire", comment rouvrir «l'horizon temporel» pour «restaurer une perspective politique» dans notre présentisme apocalyptique? Sauf à basculer dans une utopie scientiste et postpolitique à la Houellebecq, ces fictions de la fin du monde cherchent une réponse au nihilisme. Car le nihilisme est la «dissimulation du péril» et non son imagination inquiète, la «dénégation du délai» et non son orchestration littéraire.

En 2012, tandis qu'un déluge de kitsch ou de camp (post)apocalyptique se déversait sur les toiles, un double propos critique s'est fait entendre qui, non réductible à l'idéologie en question, jetait sur cette culture une lumière ironique, mais de deux manières fort diffé- rentes, et même antinomiques: celle d'un philosophe, Michaël Foessel, et celle d'un écrivain, Michaël Ferrier. L'un enseigne la philosophie à l'École polytechnique et à l'Institut catholique, l'autre enseigne la littérature à l'université Chuo de Tokyo. Le premier, né en 1974, est spécialiste de Kant et de Ricœur, et on a pu le lire dans Esprit et Libération sur des sujets d'éthique sociale (suicide en entreprise, euthanasie, captation de la «vie» et de «l'art» dans le discours managérial). Le second, né en 1967, est essayiste, romancier et critique ; $\mathrm{d}$ 'ascendance indienne et mauricienne, il est un virtuose de l'interculturel, créolisant le japonais et le français ${ }^{2}$. Il est à présent consacré comme $l^{\prime}$ «écrivain sismographe» de Fukushima, récit d'un désastre (Gallimard, 2012), coauteur avec Kenichi Watanabe du film Le Monde après Fukushima ${ }^{3}$. Son œuvre vient nourrir la littérature de «l'après » - et plus singulièrement des catastrophes nucléaires, qui, depuis Tchernobyl, ne se limitent plus à la «littérature de la bombe».

Ces deux livres font désormais partie de la culture apocalyptique en son versant critique. Mais c'est pour des raisons plus précises qu'ils se prêtent au parallèle, et même à la confrontation. On pourrait mener celle-ci sur plusieurs plans à la fois. L'essentiel ici est ce que chacun, face à l'idée de fin du monde ou à la fin effective d'un monde, entreprend de «sauver» et de penser du monde à venir.

\section{Contrepoint}

D'un côté, donc, un ouvrage de philosophie politique et morale, de l'autre un témoignage littéraire sur Fukushima. Ces deux livres sont aussi étrangers l'un à l'autre par leur visée que par leur composition. Dans Après la fin du monde. Critique de la raison apocalyptique (Seuil, 2012), Foessel s'est placé dans le sillage intem- 
pestif du criticisme kantien. Il déconstruit «l'hyperbole» de la fin du monde comme «erreur de perspective» et invoque un nouveau cosmopolitisme, à l'issue d'une démonstration en deux temps: une «Généalogie» des présupposés du catastrophisme propre à la «raison moderne» (1), puis un «Diagnostic» des expériences contemporaines de "perte en monde", analysées comme des formes $\mathrm{d}^{\prime}$ " errance » et de "suspens» (2). Le monde peut se perdre, mais pas se terminer: il sera transformé. Tout le livre est un plaidoyer pour le «monde» comme horizon de possibles nés de sa contingence et de l'indéfinition humaine. "C'est parce que le monde peut être perdu qu'il doit devenir l'objet d'une sollicitude politique", écrit Foessel (p. 161). Là où l'acosmisme apocalyptique engendre un sauve-qui-peut-la-vie, il faut agir dans le monde pour que de telles expériences de perte en monde soient impossibles.

C'est en réinvestissant la notion de «monde» contre celle de «vie» que Foessel rompt avec l'idée de "fin du monde", opposant une technique d'euphémisation rationnelle à «l'hyperbole» de la fin, ramenée à une structure irrévocable de l'entendement, due à notre désir d'éternité: la «fin du monde» est ainsi reconnue et neutralisée. La «fin de l'histoire» n'est pas davantage une expérience réelle. De même que l'être humain ne doit pas être réduit à sa vie, le monde ne doit pas être identifié à l'histoire, qui elle-même n'a rien d'un "processus» inéluctable. Si la globalisation actuelle semble par bien des traits relever d'un tel processus, entériner sa fin serait céder au fatalisme ou revenir à l'idée de nécessité historique. Foessel lui oppose l'historicité selon Heidegger: comme le disent les ruines d'une époque passée, l'histori- cité d'un objet n'est autre que son appartenance au monde, et l'histoire humaine est une suite de mondes. Le monde n'est pas l'ensemble des choses mais ce qui leur assigne un sens et permet d'en user («mundus»: «propre à»). Foessel oppose à la philosophie de l'histoire une philosophie du monde ouvert à l'histoire, fidèle à son inachèvement constitutif: le monde comme «transcendance qui reste».

Dans Fukushima, Michaël Ferrier cherche, lui, comment écrire ce qu'il a $v u$, entendu, éprouvé au Japon, entre le jour où la terre s'est mise à trembler, le 11 mars 2011, et celui où il est revenu d'un périple éprouvant, mais délibéré: en «montant» vers la «zone interdite», il a effectué une sorte de descente aux enfers avec Jun, sa compagne. Le «récit d'un désastre» est celui de trois cataclysmes en chaîne: un tremblement de terre de magnitude 9, un tsunami d'une violence exceptionnelle, et une explosion nucléaire d'une ampleur inédite - le dégagement de césium 137, rappelle-t-il, a été 168 fois plus élevé qu'à Hiroshima. Devant cette "cascade de catastrophes» aux allures d'apocalypse, Ferrier décrit et raconte, mais aussi écoute, recueille, note et annote, les yeux braqués sur les signes et les traces de ce «désastre démesuré», qui fait signe à tous au-delà d'un pays sinistré. Il se débat, lui, avec un monde de débris, et ces débris, dit-il, sont le contraire même de la «ruine romantique».

$\mathrm{Au}$ fil du texte, cette triple expérience se transforme en un immense point d'interrogation posé sur nos «manières de vivre» présentes et à venir. "La demi-vie, mode d'emploi», ainsi s'intitule l'inquiétant dernier chapitre relatif à la catastrophe nucléaire. Témoin lui- 
même, Ferrier recueille un grand nombre de témoignages: survivants, objets et paysages eux-mêmes rescapés. Se déplaçant dans l'espace japonais, il explore aussi le temps long des séismes et celui de leurs chroniques (japonaises, mais aussi chinoises), cherchant des modèles pour le «tremblement» et la «vibration» nécessaires. Voyant un univers s'effondrer, mais aussi une société résister, il note le travail énigmatique d'une beauté qui s'obstine, celle des arbres qui imperturbablement fleurissent. Il observe les survivants résister au désespoir par des gestes minutieux, cherchant d'où leur vient leur «aplomb» parfois sidérant. Il cherche une écriture du désastre qui résiste au désastre, et il en trouve le modèle un jour dans le paysage: les 260 petites îles dispersées du site de Matsushima ont mieux résisté à la vague que les grandes digues et enceintes d'Higashi, il faut donc écrire "par îlots ou par estuaires, par petites notes déferlantes... » (p. 143).
Ce qui s'apprend là d'un monde explosé, puis sinistré, nécessite pour l'écrivain un désapprentissage, qui touche aux grands écarts interculturels. Le livre, gorgé de littérature et de poésies ancienne et moderne, occidentale et extrême-orientale, dit l'événement au prisme d'une mémoire européenne et française (Pinguet et Claudel, Céline et Rimbaud), mais plus encore à travers celle du Japon ancien et moderne, quand la terre grondait et tremblait déjà et qu'un monde finissait pour de vrai: le Dit des Heike, qui narrait la fin d'une dynastie et le début d'une autre, parcourt le récit, qui s'achève avec les Notes de Hiroshima de Kenzaburo Ôe. Mais ce désapprentissage touche à une expérience plus radicale: celle de l'écrivain qui tombe sous la table et des bibliothèques qui s'effondrent - à commencer par la sienne, où seul Baudelaire est resté en place. Baudelaire, à qui Foessel a confié sa toute première épigraphe, «Le monde va finir» (Fusées).

\section{Un plaidoyer pour le "monde» contre la «fin du monde ». Questions à Michaël Foessel}

Mais la modernité dont parle Michaël Foessel est celle qui débute avec l'humanisme au moment de l'effondrement du «cosmos» (ordre du monde ou création providentielle) et se poursuit avec les Lumières. Foessel suit les différentes réponses philosophiques à cet ébranlement: d'un côté, la «voie métaphysique», des formes de pensée «acosmique» s'efforçant de se dégager de la contingence en affirmant un absolu («Dieu ou la nature » chezSpinoza, l'esprit chez Hegel, la vie chez Bergson), de l'autre, un inves- tissement du «monde» sous le signe de la contingence, et cette "voie critique» commence avec Hobbes. Au «citoyen» de Hobbes, à «l'habitant du monde» de Kant, Foessel ajoute «l'horizon» de Husserl, «l'être au monde» de Heidegger et le «monde» selon Arendt, tout en citant Bourdieu sur les raisons sociales du millénarisme et Weber sur "l'ascétisme» capitaliste. Cette construction est destinée à ruiner l'alarmisme catastrophiste, propice depuis toujours aux manipulations politiques, et à réfuter sa version 
«éclairée», jugée impropre à lutter contre les effets aliénants ou dévastateurs de la globalisation. Traitant des expériences effectives de "perte en monde», Foessel s'arrête sur deux exemples: d'une part, Jan Karski échouant à décrire le ghetto de Varsovie dans Shoah de Lanzmann («Ce n'était pas un monde», «Je n'appartenais pas à cela »), d'autre part, dans le film Allemagne année zéro de Rossellini, l'épisode où le jeune Anton se jette dans le vide après avoir erré dans la ville en ruine, relu par Deleuze (L'Image-temps). À ces moments, le monde cesse d'être habitable, car la rupture entre l'action et le sens le détruit comme «horizon de significativité».

La perspective anti-apocalypticienne de Foessel est intimement liée à son régime de rationalité et à sa pensée du réel, sensible autant dans son ton que dans sa composition. Les deux parties du livre sont rythmées par quatre «intermèdes» qui évoquent, par associations et montage, des œuvres littéraires et cinématographiques: glissée dans les intermèdes et les épigraphes, la réalité des oscillations et contradictions propres aux œuvres d'art trouve place comme au creux de cette "critique de la raison apocalyptique».

Celle-ci s'amorce d'ailleurs avec une critique de «l'image du monde» en tant qu'image. Et l'image est ici ramenée à une condition de l'entendement, non à une forme productrice et créatrice de pensée (comme elle l'est chez Hans Blumenberg). C'est à la philosophie seule qu'est confié l'art de se dégager de l'alternative entre catastrophisme et optimisme: «La philosophie peut par ses propres moyens produire une critique de la raison catastrophiste sans céder à un quelconque optimisme.» Disant cela, Foessel prend ses distances surtout avec les «batailles d'experts» sur les probabilités de la catastrophe, climatique ou autre. Il renonce à traiter du contenu du débat pour se concentrer sur les «termes du débat». Le savoir scientifique est traité en obstacle à contourner, et même en repoussoir, elliptiquement identifié à sa manipulation politique.

\section{Le «monde " et la «vie ». Poétique et politique}

Les deux livres exercent une certaine critique de la culture apocalyptique. Ferrier écrit contre la puissance de fascination des images médiatiques, constitutivement étrangères au réel, qui amalgament les désastres et se répètent en boucle, nourrissant une «jubilation dans le pire».

Contrairement à ce qu'on a pu lire ou entendre ici ou là, Fukushima n'est pas une apocalypse. Ce n'est pas un accident total (encore que celui-ci soit toujours possible). Mais d'une certaine manière le pire a déjà eu lieu, il est là, tout autour de nous. C'est une catastrophe en gargouillis, non en apothéose. Une sorte de dégringolade quotidienne, systématique. Ce n'est pas une extermination violente, c'est un état létal - et désormais de plus en plus légal [...], une forme dévitalisée de la vie. (p. 249)

C'est tout autrement que Foessel critique la «logique du pire»: celle qui se 
réclame de la catastrophe pour gérer les existences et gouverner, mais celle aussi qui tire une philosophie de la vie d'une «heuristique de la peur». Actualisant le futur, cette philosophie prétend installer la fin du monde dans le domaine de la rationalité, et confie à la politique la tâche de protéger l'espèce, défigurant ainsi l'espèce et la politique. Là où Dupuy, après Anders et Jonas, font de la certitude du pire un appel à dépasser la valorisation moderne du doute, devenu dangereux et même criminel, Foessel au contraire remonte aux "Temps modernes» afin de déconstruire cette logique du pire à l'aide des esprits lucides qui s'y étaient employés alors, par une rationalité critique ou politique. C'est donc essentiellement en tant que résurgence que Foessel affronte l'apocalyptisme contemporain: le retour du motif de la fin du monde, à propos des camps et d'Hiroshima, puis aujourd'hui sur le mode écologique, réactualise des angoisses anciennes, et rejoue la destruction moderne du cosmos. Notre époque est certes "pauvre en monde», et il y a bien des expériences de "perte en monde", que les anthropologues étudient - mais de ces études Foessel ne dit rien. Un tel empirisme n'a pas sa place dans son criticisme, et s'il y prêtait oreille il lui faudrait renoncer à sa notion aseptisée de «monde», comme à sa vision de l'histoire. Son propos sur Hiroshima ne s'embarrasse pas plus de témoignages internes que sur le ghetto de Varsovie. Foessel bataille contre une idée du temps pour établir une idée du monde, mais il n'envisage pas qu'un savoir concret sur notre monde soit nécessaire pour penser notre temps.

C'est ici que le contrepoint des deux livres devient précieux. Le "récit d'un désastre» de Ferrier semble être le précis implacable et sensible d'une de ces "pertes en monde» dont Foessel ne dit rien de concret - sauf à revenir au nazisme et à ses ruines, là encore sur un mode euphémistique («l'errance» de Karski et d'Anton).

Là où le texte de Foessel se veut d'emblée politique en opposant le «monde» comme catégorie politique arendtienne à l'injonction de "préserver la vie», le texte de Ferrier devient le plus profondément politique au moment d'évoquer l'altération qu'inflige à la vie de populations entières le choix civilisationnel de l'industrie atomique. Les maisons se réparent, mais «qui va réparer la mer?» demande un pêcheur. Ferrier ne dissocie jamais la description du monde d'une interrogation sur la vie et inversement $-c^{\prime}$ est là du reste sans doute le propre d'une œuvre littéraire, qu'elle relève ou non du témoignage. La défiguration de l'un entraîne celle de l'autre. «La vraie vie est ailleurs et nous ne sommes pas au monde»: cette formule de Rimbaud, citée par Ferrier à la fin de son livre, donne tout leur sens aux considérations terribles sur la «demi-vie» qui s'est installée "après», contaminant un pays, mais aussi, moralement, une civilisation entière. Ce terme de «demi-vie», détourné du lexique scientifique où il désigne la durée de radioactivité de chaque substance nucléaire - et ces durées sont proprement vertigineuses -, vient questionner l'ensemble de nos conduites, de même que l'ébranlement du séisme et le tohubohu du tsunami bouleversaient tous les points de repère de la vie commune et les modes de perception sensorielle.

Des populations entières réduites au silence et à la résignation, des rejets chroniques et continuels tolérés et même homologués, des déchets intraitables qu'on transmet, toute honte bue, 
à ceux qui viendront après. Et cette furie se propage le plus tranquillement du monde. C'est la «demi-vie». Insaisissable, impalpable, nébuleuse et irréfutable à la fois, subreptice et pourtant éclatante dans la limaille des jours, la demi-vie s'impose comme le seul modèle de nos économies et de nos modes d'existence.

Ce modèle qui altère la vie sur terre et sur mer menace bien le monde comme habitat et entité à la fois. Car ce n'est évidemment pas de la seule vie biologique qu'il s'agitici, ni de l'élan vital, ni de la seule bonne santé de la planète Terre, mais de nos «modes d'existence» et «manières de vivre», c'està-dire d'habiter cette Terre: il y va bien là de nos manières d'user du monde, mais aussi de le regarder et de l'aimer, il y va de la possibilité, mais aussi de la beauté de la vie, de nos formes de vie qui donnent forme au monde. Rivé au biopolitique, sourd au concept de forme de vie bien que citant Foucault et parfois Agamben (pour critiquer Le Règne et la Gloire), Foessel semble devoir rester étranger même à la question commune de l'art de vivre, alors pourtant qu'il parle un moment de «la vie sous condition de monde». Quant à Ferrier, c'est à Guy Debord qu'il donne le dernier mot politique, citant un texte sur «la dernière doctrine de la sécurité des centrales nucléaires » paru en 1988 dans ses Commentaires sur la société du spectacle, texte dont Fukushima confirme selon lui la pleine et littérale actualité. Debord résumait ainsi la doctrine: "Puisque l'accident est toujours possible, ce qu'il faut éviter, c'est qu'il atteigne un seuil catastrophique, et c'est aisé. Il suffit de contaminer coup par coup avec modération.» Fukushima. Récit d'un désastre construit sa courbe politico-poétique entre cette quasiprophétie de Debord et les Notes de Hiroshima de Kenzaburo Ôe (1965), référence qui semble couronner l'inquiète poétique de la notation à laquelle s'essaie ce récit. Ôe avait perçu en effet l'énormité du problème qu'engendrait le manque de mots pour dire la réalité de la catastrophe nucléaire: tout bonnement la disparition de la réalité. Cette réalité, Ferrier la résume ainsi:

La trajectoire de cette pâte incandescente hautement radioactive, qui a percé depuis longtemps les cuves des réacteurs et se dirige on ne sait où dans le sous-sol, est pourtant tout simplement - et sans céder à aucun catastrophisme le problème le plus grave auquel l'humanité ait jamais été confrontée.

C'est cette gravité-là, étrangère au «catastrophisme» mais menaçant le monde, que la philosophie livrée par Foessel à ses «propres moyens » renonce à intégrer.

\section{Croire au monde}

À Fukushima le monde n'est pas détruit: il vacille et semble s'éclipser, clignoter. Ce vacillement fait acquérir un autre savoir sur le monde:
On apprend que le monde n'est pas un terrain solide et stationnaire, réglé en fonction d'une norme permanente et dominante, mais une formation en 
vibration continue. On se retrouve seul, les sens aiguisés, dans un univers multiforme de sons et d'objets, d'odeurs, de goûts et de corps, tous soudain retrouvés dans l'immédiateté du réel, sa précision absolue. (p. 54)

C'est à cet aiguisement des sens que travaille l'auteur, et cette "immédiateté du réel » se cherche dans une forme capable non seulement de décrire un paysage inédit qui n'est plus «lisible» (p. 182), mais de traduire l'expérience du temps, en mutation continue au cours des trois catastrophes. Imaginant un art inouï de la notation accélérée, l'auteur cite une série de chroniques de séismes, dont les Notes éparses sur le grand tremblement de terre de 1923 d'Akutagawa, écrites lors d'un séisme plus grave encore. Mais avec la «demi-vie», la dimension même du temps devient une expérience nouvelle, ou plutôt une sensation: celle d'être enfermé dans une prison mobile. Et c'est par l'image, le son et le rythme qu'il tente de dessiner les contours durables d'une éthique à hauteur des gestes et des formes d'existence qu'il voit s'improviser tout au long du voyage.

L'auteur note les dégâts du séisme, mais il enregistre aussi sa puissance de révélateur sur les individus, qui semblent tous réapparaître au monde - parfois dans des grimaces et des contorsions de douleur, parfois aussi dans une grâce inconnue. Alors que tout s'est effondré des maisons, des bibliothèques, des villes et des existences, alors que les ravages du tsunami ont produit un paysage illisible, un monde qui cesse d'être monde, alors que le village frontalier de la zone interdite a fait place à une lugubre ville fantôme dans un paysage déserté par les hommes - on n'y entend plus que des plaintes d'animaux de loin en loin -, çà et là émerge un chant ou même un rire qui vient "sauver» quelque chose du monde. Le texte semble alors retrouver l'apocalypse comme récit véridictionnel, mais en substituant au salut un sauvetage de fortune, adéquat à ce monde de «débris». Recueillant les récits des témoins, Ferrier observe leurs visages et rassemble les signes d'une force mentale que métaphorise le «manche de l'éventail» (ôgi no kaname): le "manche», c'est en réalité son "pivot», par quoi l'éventail peut vibrer, s'ouvrir et se fermer. L'auteur précise que cet endroit unique, qui reste immobile au milieu du séisme, n'a rien d'une "transcendance»: c'est juste un point précis dans un bout de bois.

Sans le vouloir, le livre de Ferrier semble ainsi répondre presque point par point à celui de Foessel, ou plutôt l'interroger radicalement quant à sa méthode. Là où Foessel tente de reconstruire méthodiquement le concept de «monde» pour le reconduire à l'espoir politique contre la "mondialisation», Ferrier, décrivant la vie sinistrée sous l'angle de la "vraie vie» absente, renoue avec ce qu'Arendt et Deleuze avaient tous deux, dans deux périodes très différentes, appelé la "croyance au monde». «Les seuls à croire encore au monde sont les écrivains et les artistes ", écrivait Arendt au cours des années cinquante dans les brouillons de Qu'est-ce que la politique?. "Il faut que le cinéma filme, non pas le monde, mais la croyance à ce monde, notre seul lien», disait Deleuze en 1985 dans L'Image-temps. Cette tâche était encore politique, alors que l'espoir d'une transformation $\mathrm{du}$ monde par le cinéma tenait de la "déclaration de musée». "Croire, non pas à un monde meilleur, mais au lien de l'homme et du 


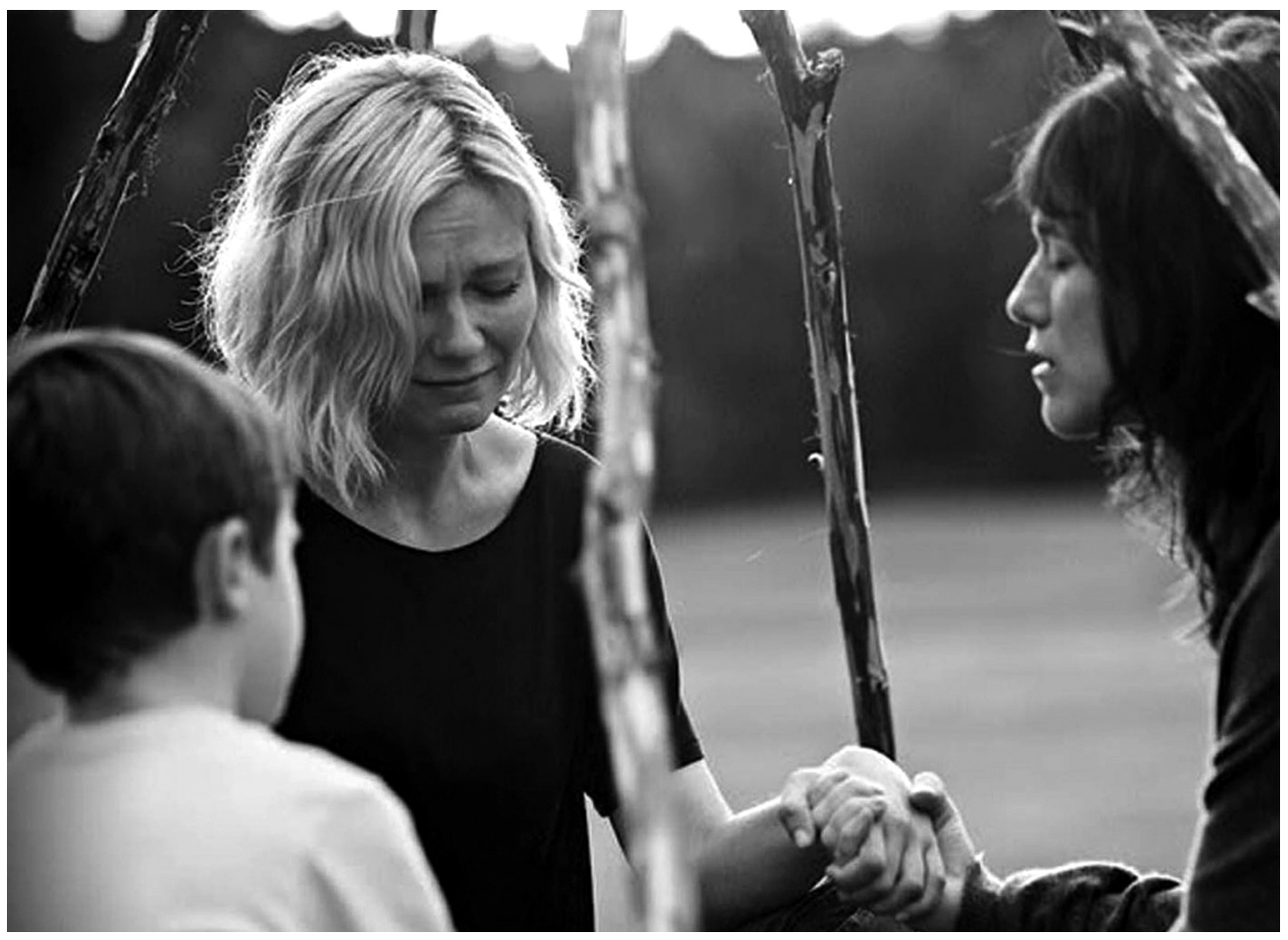

Melancholia (Lars von Trier, 2011)

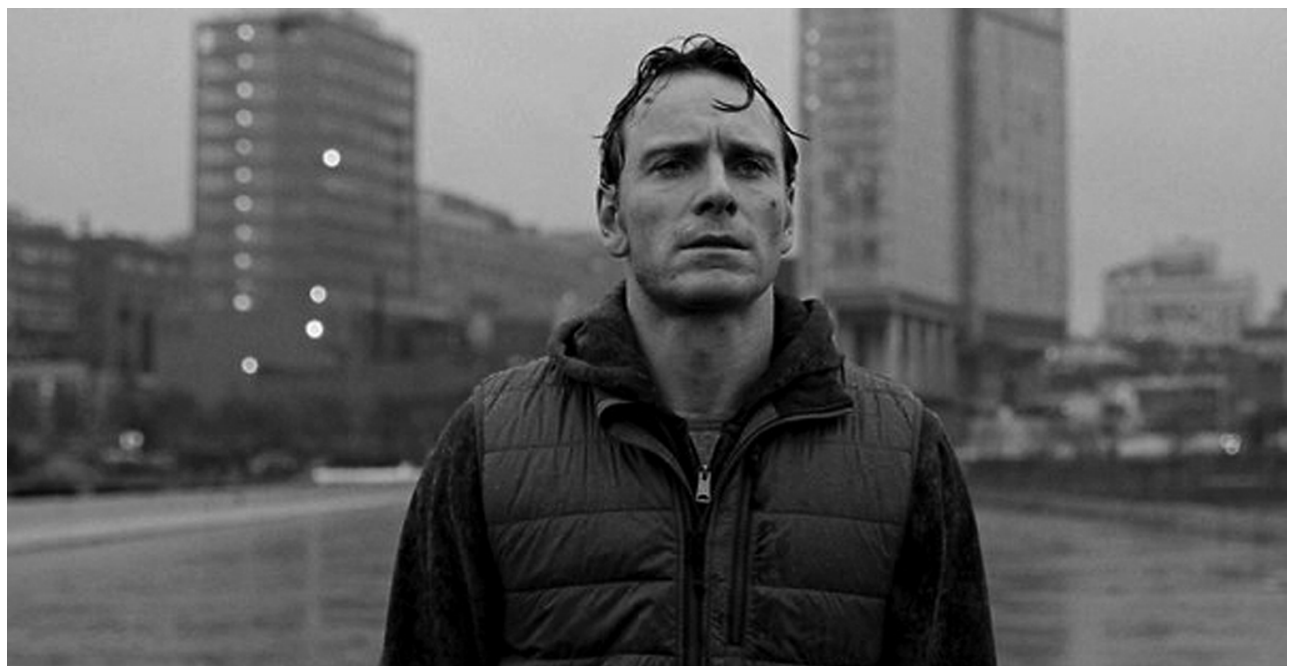

Shame (Steve McQuenn, 2011)

monde, à l'amour ou à la vie, y croire comme à l'impossible, à l'impensable, qui pourtant ne peut être que pensé: "Du possible, sinon j'étouffe." "
Ce possible-là n'est pas celui dont parle Foessel, qui, citant ces lignes, les révoque: le «suspens» deleuzien reste «contemplatif» et «métaphysique». Deleuze est 
coupable de croire en la « vie » à la manière de Bergson et des philosophes de l'expérience, pour qui «croire est une opération de la vie destinée à s'assurer elle-même». Mais quand Foessel, en «intermède», raconte comment Brandon, dans Shame de Steve McQueen, doit prendre sa sœur suicidaire dans ses bras et se salir de son sang, ou comment Justine, dans Melancholia de Lars von Trier, "fait un monde" pour quelques minutes avant l'explosion

\section{Notes}

1 Jean-Paul EngÉLIBERT, Apocalypses sans royaumes. Politique des fictions de la fin $d u$ monde, $\mathrm{XX}^{e}-$ XxI siècles, Classiques Garnier (Littérature, Histoire, Politique), 2013. Voir dans ce volume sa lecture par Dominique Rabaté, p. 206-208.

2 Dans un entretien avec Dany Laferrière paru dans la NRF en 2012, Ferrier s'est dit «écrivain en tenant les mains à sa sœur et son neveu sous leur "cabane magique», fait-il autre chose que croire à son tour - ici non plus au monde, ni à la vie, mais à l'amour, qui n'est lui aussi qu'une «opération destinée à s'assurer elle-même»? Le philosophe semble même croire alors à une fatalité salutaire: "Il y a une certaine fatalité à ce que, dans une époque pauvre en monde, le rappel de l'expérience incombe à des insensés» (p 198). japonais», et il a écrit (en anglais) sur le «Japon créole» ("Creole Japan; or, The Vagaries of Creolization», Small Axe, vol. 14, no 3 33, 2010, p. 33-44).

3 Ce film (77 min, 2012) a obtenu en 2013 le prix Lucien Kimitété du Festival international du film insulaire de Groix. 